\title{
Quantitative Analysis of Phase Trajectory as the Information about Technical Condition of the Object
}

\author{
L. MAJKUT* \\ AGH University of Science and Technology, Faculty of Mechanical Engineering and Robotics \\ Department of Mechanics and Vibroacoustics, al. A. Mickiewicza 30, 30-059 Krakow, Poland
}

\begin{abstract}
The paper aims to describe the potential use of phase trajectory for damage detection of structural components. The attractor of the examined trajectory is a static equilibrium point of the element being diagnosed. Two different damage indices are proposed to evaluate the severity of damage in the diagnosed component. The first one refers to distance between a point on a trajectory and the point which is the attractor of the trajectory. The other one relates to linearity of the Poincaré map. Effectiveness of the proposed method was evaluated on: a simple model with two degrees of freedom, data from the finite element method model of a cantilever beam with a crack, and experimental data for a cracked plate. With the proposed method, the damage can be quickly and effectively detected. By comparing the current trajectory with that from the previous diagnostic test, it is possible to establish if the nature of crack is either propagating or stationary.
\end{abstract}

PACS: 43.40.Le, 46.40.-f

\section{Introduction}

Owners and users of building, mechanical, aviation, hydro-engineering or cosmic structures take every effort to prolong the lifetime of such structures, and maintain the operating safety at the same time. On the other hand, the safety of numerous systems is crucial: damage of structures (building, planes, bridges or power facilities) would have disastrous consequences in terms of the number of casualties, extent of environmental harm or property loss.

Defects (faults, damage) are unavoidable in materials and structures, and their presence can reduce the mass, rigidity, strength and safety of such structures. When such structures are in service, the fault raises to the critical values, leading to disintegration of a component in the end.

On the one hand, occurrence of a macrocrack causes discontinuity of the material, which can be detected with ultrasonic, magnetic and eddy current testing or, in most cases, by visual inspection [1]. On the other hand, the faults change the physical properties of the structure being studied and their appearance is reflected in variations of their dynamic characteristics. Such property is the basis for vibroacoustic diagnostics which allows to detect local faults by measuring global values. Symptoms of damage are sought in variations of, among others: frequency of the natural vibration [2-8], modal shape [9-14], damping coefficients $[15,16]$, forced vibration amplitudes

* e-mail: majkut@agh.edu.pl
[17-22], antiresonance frequencies [23, 24], higher order statistics [25-28] or scaleogram (wavelet transform) [29-31].

Observation and analysis of vibroacoustic processes can be used for detection, localization and quantification of damage in locations which are difficult to access or even inaccessible [32], and with the possibility to detect such damage quickly and at relatively low cost, the vibroacoustic method has become an effective and frequently used nondestructive testing (NDT) technique applied to determine the technical condition of structures.

The present paper aims to describe the potential use of phase trajectory, the attractor of which is a static equilibrium point of the element being diagnosed (static deflection of the component).

The effectiveness of the proposed method was, at first, tested on a simple model with two degrees of freedom (dofs). The damage was modelled by simultaneous changing the stiffness and damping coefficients connecting the dofs. Two different damage indices are proposed to assess the severity of damage.

After testing the effectiveness of the proposed method on a simple model with two dofs, it was tested with the finite element method (FEM) model data of a cantilever beam with a crack and using experimental data recorded for an aluminium plate cracked across the material.

With the diagnostic method based on quantitative analysis of the phase trajectory, the damage can be detected quickly and effectively. By comparison the current trajectory with that of the previous diagnostic test, it is possible to establish if the nature of crack is either propagating or stationary. 


\section{System dynamics in the phase space}

Phase space of a dynamic system is the mathematical space of orthogonal coordinates representing all variables essential to determine temporary state of the system. Each dynamic system can be described with a system of differential equations:

$$
\frac{\mathrm{d} \boldsymbol{x}}{\mathrm{d} t}=f(\boldsymbol{x}(t))+\boldsymbol{u}(t),
$$

where $\boldsymbol{x}$ - state vector, $\boldsymbol{u}(t)$ - input (forcing) vector.

Unfortunately, for the most of dynamic systems, complete information regarding the velocity and displacement of all points (degrees of freedom) is not available.

Exhaustive description of the system in phase space can be obtained also if system attractors are known. The attractor is a certain set in the phase space to which trajectories beginning in various areas of the phase space evolve after a long enough time, i.e. trajectories for various initial conditions.

The attractor can be a point, a closed curve (limit cycle), a manifold (e.g. limit torus) or a fractal (strange attractor). Attractor is one of the key terms used in chaos theory [33]. Each attractor has its attraction area, known as the attraction basin (a set of such initial conditions for which the trajectory evolves to the attractor).

The potential use of the limit cycle quantitative analysis for a structural components diagnostics has been described in other papers of the present author, e.g. [34-36] and Trendafilova [37]. The present paper focuses on the potential uses of the quantitative analysis of a trajectory in case when the attractor is a point.

Phase trajectory in phase space is a multidimensional curve (each degree of freedom is represented as its separate dimension). With no harm to the general nature of consideration, trajectory projection on certain plane, formed by two perpendicular axes of the phase space, can be analysed $[33,38]$.

In the next section various coordinates of axes forming the projection plane for the phase trajectory are described. In other words, those are the variables (e.g. acceleration, velocity, displacement) of one point of the component or structure being diagnosed, which can be used for determining the trajectory projection plane.

\section{Methods for determining trajectory}

The most apparent coordinates of the plane on which the phase trajectory can be projected are used for topological analysis of vibrations: these are the velocity and displacement. Determining a trajectory on such plane involves the determination with an accelerometer, a time series of vibration acceleration and integrating it afterwards. Each point on the trajectory has the coordinates corresponding to displacement and velocity values on the horizontal and vertical axis, respectively. The values are determined in the same moment of time.

In lieu of velocity as the function of displacement, the acceleration as the function of displacement can be analysed or the acceleration as the function of velocity. In addition to obvious benefits from a smaller number of the time series integration (time and cost of calculations), another advantage of the trajectory of the velocity-acceleration coordinates is that it can be determined by measuring directly on the tested structure. To carry out the tests, the VS80 Brüel \& Kjær velocity sensor and PCB 356A16 PCB Piezotronics accelerometer were used.

Other method for constructing projection plane, known from chaos theory, is the use of the method of delays. Using that method, it is sufficient to determine one time series (e.g. vibration acceleration) and, based on that, determine the phase space including the plane sought, on which the phase trajectory is projected.

The reconstruction of phase space is based on the fact that the time series contains some information of non-observed states of the system generating it (component being diagnosed) and dynamics of such states.

Reconstruction of a trajectory based on a single time series requires formation of additional variables. The Takens theorem [38] is useful while seeking new variables, whereby each point in the phase space $a(n)$ is represented by a series of subsequent values of the time series

$$
\begin{aligned}
& a(n)=[y(n), y(n+\tau), \ldots, y(n+(m-1) \tau)], \\
& a(n+1)=[y(n+1), y(n+1+\tau), \\
& \quad \ldots, y(n+1+(m-1) \tau)],
\end{aligned}
$$

where $m$ - dimension of the phase space, $\tau$ - time delay.

Reconstruction of the space with the method of delays requires its dimensional parameter $m$ and the time delay $\tau$ to be adopted a priori.

The most commonly used procedure for determination of the space dimension is the false nearest neighbours method. The method is based on the fact that for sufficiently large $m$, in the reconstructed trajectory the proportions of distance between states no longer change significantly i.e. close states in $m$-dimensional space remain close, also after the addition of $m+1$ coordinate 390].

Due to the use (for diagnostics purposes) only the projection of as-reconstructed trajectory on the plane, determined by the coordinates, e.g. $y(n)$ and $y(n+\tau)$, the knowledge of the dimension of phase space is dispensable.

The value of the time delay $\tau$ can be determined as a result of the autocorrelation function analysis, as the moment in which the function gets the zero value for the first time. A major drawback of the autocorrelation based method is an assumption of linear dependences between observations [40]. The criterion for selection of delay, which also uses non-linear dependences, is the mutual information method. The amount of mutual information $I\left(x_{i}, T\right)$, is obtained from the equation [40]:

$$
\begin{gathered}
I\left(x_{i}, T\right)=\frac{1}{N} \sum_{n}\left[\log _{2}\left(p\left(x_{i}(n), x_{i}(n+T)\right)\right)\right. \\
\left.-\log _{2}\left(p\left(x_{i}(n)\right)\right)-\log _{2}\left(p\left(x_{i}(n+T)\right)\right)\right],
\end{gathered}
$$


where $p\left(x_{i}(n)\right)$ is the marginal probability distribution function of the analysed series, $T$ is time delay, $p\left(x_{i}(n), x_{i}(n+T)\right)$ is the joint probability distribution function, $N$ - number of samples in a time series.

According to that method, as the value of time delay $\tau$ the lowest $T$ value in Eq. (3) should be assumed, for which the function of mutual information has the local minimum.

The method of delays is the most popular, but not the only one, used for reconstruction of phase space. The most commonly known methods are: factor analysis [41], singular spectrum approach (SSA) [42] and the Pakard et al. method of derivatives [43].

\section{Quantitative analysis of the phase trajectory}

This section of the paper describes the potential use of phase trajectories, for damage detection of structural components, the attractor of which is a static equilibrium point of the component being diagnosed (static deflection of the component). In other words, it is the trajectory of the mechanical system of "reaching" the equilibrium state (point) after change of the initial conditions or after its impulse excitation.

To move to the quantitative analysis of the phase trajectory, repeatability of initial conditions and pulse excitation should be ensured during vibration analysis. In case of numerical calculations, this is done by assuming zero initial conditions and exciting vibrations with an impulse of finite (but not zero) duration. In experiments, the excitation was done with a steel ball falling freely. In each case, the same initial height of the ball was assured and bouncing was eliminated.

The effectiveness of the proposed method was, at first, tested on a simple model with two dofs, as shown in Fig. 1. The damage was modelled by simultaneous, percentage variation of spring coefficient $k_{2}$ (decreasing) and damping coefficient $c_{2}$ (increasing). For such a model, selection of damage indices was proposed.

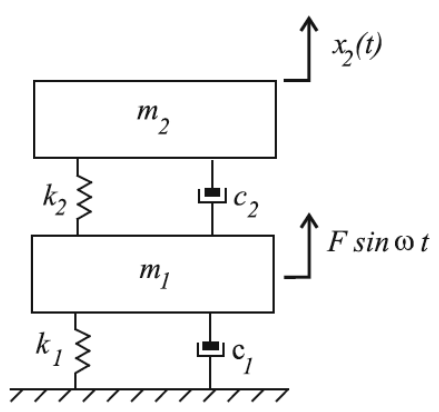

Fig. 1. System with two degrees of freedom.

Figure 2 shows an example of phase trajectories determined numerically for the damaged (continuous line) and undamaged (dashed line) system.

In Fig. 2, the initial and final parts of vibration were omitted, otherwise such samples would make the figure illegible.

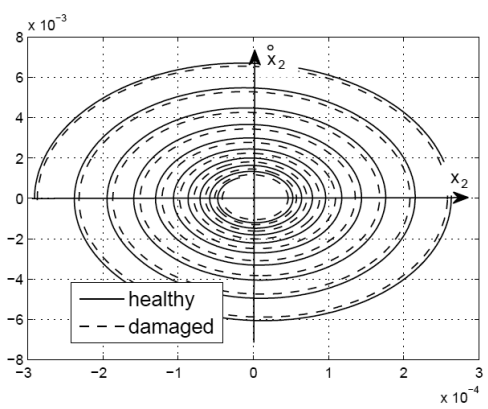

Fig. 2. Phase trajectory variation due to damage.

The author proposes to carry out quantitative analysis of the trajectory using two damage indices. The first one, refers to the distance between a point on a trajectory and the point which is the attractor of the trajectory. That is why the location of each point on the trajectory should be described by polar coordinates $(r, \phi)$.

Figure 3 shows fragments of an example of $\boldsymbol{r}(t)$ vector for an undamaged (continuous line) and damaged component (dashed line).

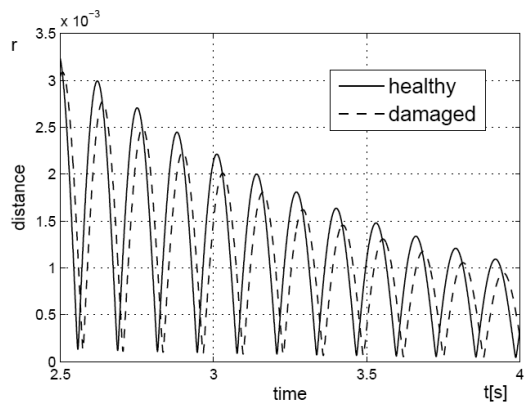

Fig. 3. Distance between the point on phase trajectory and the attractor.

As the damage index a sum of relative differences of $\boldsymbol{r}$ vectors is proposed. Such damage index $\mathrm{DI}_{r}$ is defined by the formula:

$$
\mathrm{DI}_{r}=\frac{1}{N} \sum_{n} \frac{\boldsymbol{r}_{\mathrm{d}}(n)-\boldsymbol{r}_{\mathrm{h}}(n)}{\boldsymbol{r}_{\mathrm{h}}(n)},
$$

where $\boldsymbol{r}_{\mathrm{d}}$ - vector of distance between points on trajectory and the attractor, determined for the damaged element, $\boldsymbol{r}_{\mathrm{h}}$ - vector of a healthy (or reference) element, $N$ - number of samples.

The other proposed damage index refers to the Poincaré map. The Poincaré map is a tool invented by Henri Poincaré, used for analysing phase portraits of complex systems. It is constructed by a stroboscopic "viewing" of a phase trajectory in constant intervals. In other words, the Poincaré map is composed of a series of "snapshots" of the movement in the phase space, taken in regular intervals.

Figure 4 shows an example of phase trajectory and its relevant Poincaré map. 


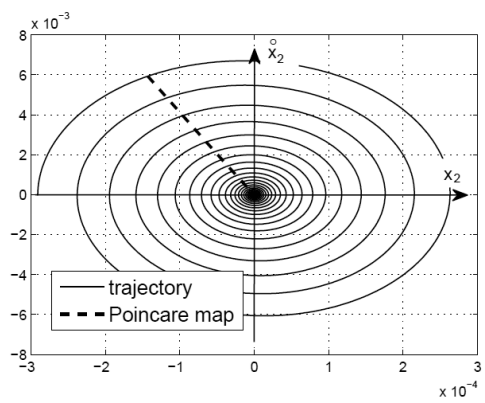

Fig. 4. Trajectory and its Poincaré map.

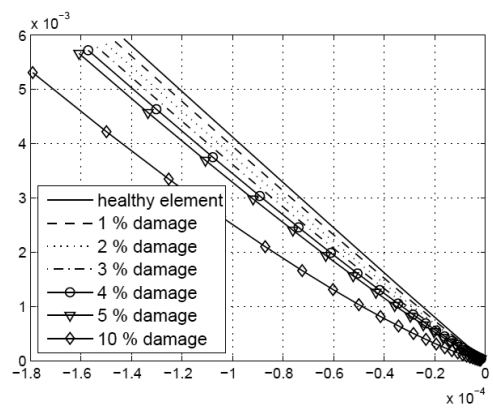

Fig. 5. Poincaré maps for the system shown in Fig. 1 with various extent of damage.

If the snapshots are taken in intervals corresponding to the period of the first natural frequency (as shown in Fig. 4), then such Poincaré map makes (after attenuation of transient processes related to higher frequencies) a straight line - points on the map aimed at the attractor. If the same intervals are used for creating Poincaré map for the trajectory of a system of other mass and stiffness parameters (e.g. damaged component), such map will no longer form a straight line.

Such "curvature" of the map is due to the fact that, as result of damage, the period of the natural vibration of the system changes, therefore the snapshots are not taken with the angle increment of a polar coordinate $\phi$ equal to $2 \pi$.

Figure 5 shows example of the Poincaré maps changes for the system shown in Fig. 1, as function of the damage severity.

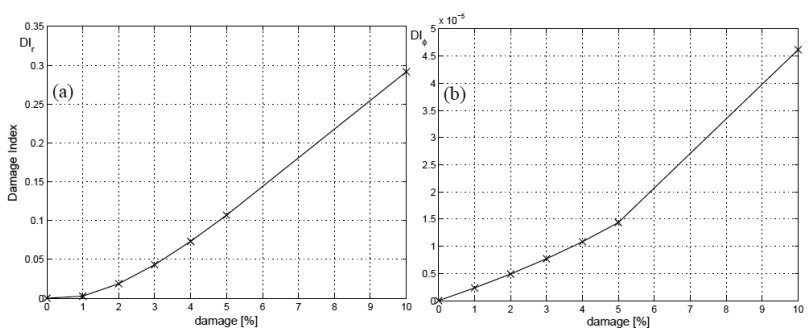

Fig. 6. Changes of the proposed damage indices as function of damage severity (a) $\mathrm{DI}_{r}$ and (b) $\mathrm{DI}_{\phi}$.
The proposed damage index involves the sum of relative differences of angle vectors $\phi$ of the Poincaré map. Such $\mathrm{DI}_{\phi}$ index is defined by:

$$
\mathrm{DI}_{\phi}=\frac{1}{N_{2}} \sum_{n} \frac{\phi_{\mathrm{d}}(n)-\phi_{\mathrm{h}}(n)}{\phi_{\mathrm{h}}(n)},
$$

where $\phi_{\mathrm{d}}$ - vector of polar coordinates of the Poincaré map determined for a damaged element, $\phi_{\mathrm{h}}$ - vector for a healthy (or reference) component, $N_{2}$ - number of samples on the Poincaré map.

Figure 6 shows the two proposed damage indices as a damage function for the two dofs system (shown in Fig. 1).

After testing the effectiveness of the proposed method on a simple model with two degrees of freedom, its effectiveness was checked on a numerical model of a cantilever beam with a crack.

\section{Analysing trajectory of a cracked beam - simulation tests}

The phase trajectory was determined by simulation of an impulse response of the beam with crack of different depth $a$ showed schematically in Fig. 7 .

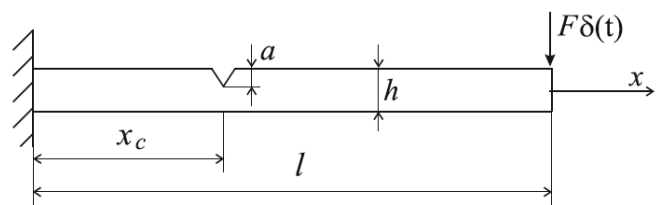

Fig. 7. Analysed beam with crack.

The beam was modelled by using one-dimensional finite elements, together with a special element with crack $[32,45,46]$. Using a one-dimensional FEM model involves determining the stiffness, mass and damping matrices of the analysed component and using them for motion equations integrated with finite difference method [47].

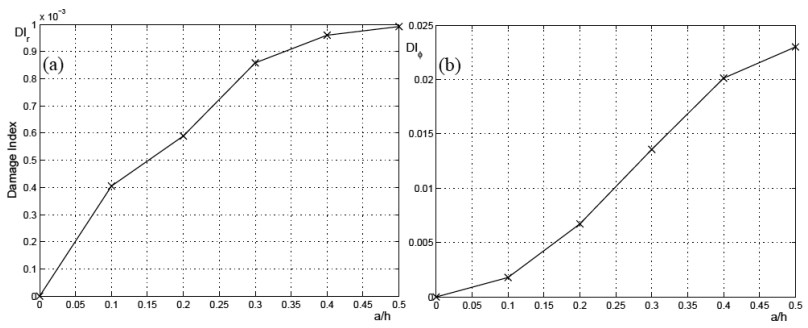

Fig. 8. Damage indices based on phase trajectory (a) $\mathrm{DI}_{r}$ and (b) $\mathrm{DI}_{\phi}$.

Figure 8 shows both proposed damage indices as the function of relative crack depth. 


\section{Analysing trajectory of a cracked plate - experimental tests}

Figure 9 shows the tested aluminium plate. For diagnostic tests 6 plates of various crack length were prepared $d=0,20,40,60,80,100 \mathrm{~mm}$. Cracks of the plate across the material were made with laser (gap width $0.4 \mathrm{~mm}$ ).

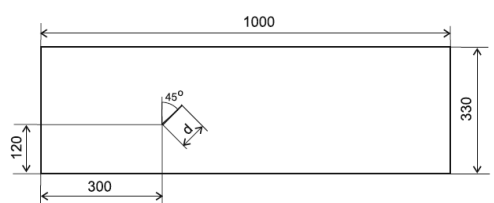

Fig. 9. Tested plate with the crack.

Phase trajectory was determined by measuring acceleration in function of vibration velocity (testing stand is shown in Fig. 10).

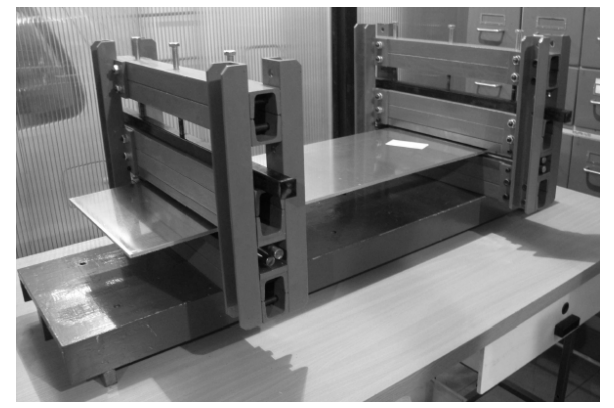

Fig. 10. Test stand.

Figure 11 shows both proposed damage indices as the function of crack length $d$.

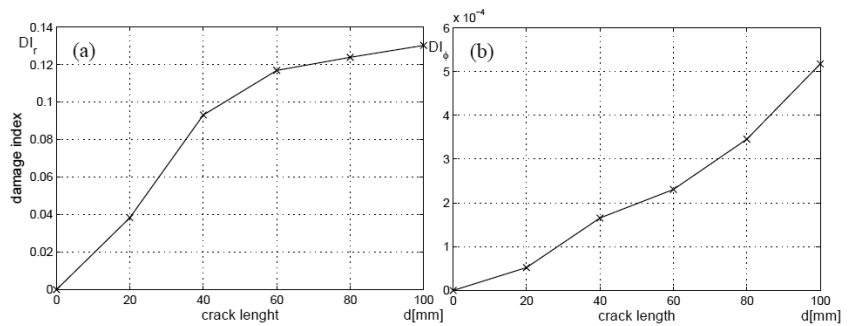

Fig. 11. Damage indices based on phase trajectory (a) $\mathrm{DI}_{r}$ and (b) $\mathrm{DI}_{\phi}$.

\section{Conclusions}

With the diagnostic method based on analysing the phase trajectory, the damage can be detected quickly and effectively.

Analysis of the damage indices as the function of damage severity shown in Figs. 8 and 11 demonstrated high sensitivity (possible detection of damage in early stage) of the proposed diagnostic method. Early detection of damage in structural component allows to: optimise repair activities (as to the scope and necessity), avoid loss related to forced downtime, reduction of the cost of undesired storage of spare parts and cost incurred through unexpected breakdowns.

High sensitivity in function of damage, involves also considerable variations of the damage index value, which allows the detection and analysis of the damage progression. In other words, by comparing the current trajectory with that of the previous diagnostic test, it is possible to establish if the nature of crack is either propagating or stationary. Only the propagating crack can cause failure of the diagnosed element.

The method does not filter non-linear effects and variations of the frequency structure in the diagnostic signals, related to the progress of damage, which can be considered as its special advantage.

Practical application of the phase trajectory based methods appear a very useful tool for identifying the process of damage initiation and propagation. It can serve as major indicator of damage and is easily adaptable in practical applications.

\section{References}

[1] A. Grandt, Fundamentals of Structural Integrity: Damage Tolerant Design and Nondestructive Evaluation, Hoboken, 2004.

[2] M. Cerri, G. Ruta, J. Sound Vibrat. 270, 39 (2004).

[3] J. Fernandez-Saez, C. Navarro, J. Sound Vibrat. 256, 17 (2002).

[4] S.K. Maiti, S.M. Wathare, Int. J. Fracture 141, 339 (2006).

[5] L. Majkut, Arch. Acoust. 31, 17 (2006).

[6] J. Lee, J. Sound Vibrat. 320, 482 (2009).

[7] L. Majkut, Latin Am. J. Solids Struct. 7, 423 (2010).

[8] L. Majkut, Latin Am. J. Solids Struct. 7, 437 (2010).

[9] J.-T. Kim, Y.-S. Ryu, H.-M. Cho, N. Stubbs, Eng. Struct. 25, 57 (2003).

[10] B. Kim, S. Yoo, S. Lee, S. Baik, Key Eng. Mater. 321-323, 1620 (2006).

[11] J. Morlier, F. Bos, P. Castera, Key Eng. Mater. 293-294, 305 (2005).

[12] S. Caddemi, I. Calin, J. Sound Vibrat. 327, 473 (2009).

[13] M. Chandrashekhar, R. Ganguli, J. Sound Vibrat. 326, 939 (2009).

[14] S.-E. Fang, R. Perera, J. Sound Vibrat. 324, 40 (2009).

[15] G. Kawiecki, Smart Mater. Struct. 10, 466 (2001).

[16] C. Kyriazoglou, B. Le Page, F. Guild, Composites Part A: Appl. Sci. Manufact. 35, 945 (2004).

[17] M. Cao, Q. Ren, P. Qiao, Key Eng. Mater. 324-325, 343 (2006).

[18] G.M. Owolabi, A.S.J. Swamidas, R. Seshadri, J. Sound Vibrat. 265, 1 (2003). 
[19] J. Lee, J. Sound Vibrat. 326, 205 (2009).

[20] J. Sawicki, M. Friswell, Z. Kulesza, A. Wroblewski, J. Lekki, J. Sound Vibrat. 330, 1365 (2011).

[21] L. Wang, Z. Yang, T.P. Waters, J. Sound Vibrat. 329, $5070(2010)$.

[22] U. Andreaus, P. Baragatti, J. Sound Vibrat. 330, 721 (2011).

[23] M. Dilena, A. Morassi, J. Sound Vibrat. 276, 195 (2004).

[24] J. Sinha, M. Friswell, Computers Structures 80, 1473 (2002).

[25] S. El-Ouafi Bahlous, H. Smaoui, S. El-Borgi, J. Sound Vibrat. 325, 49 (2009).

[26] A. Chatterjee, J. Sound Vibrat. 329, 3325 (2010).

[27] C.R.P. Courtney, S.A. Neild, P.D. Wilcox, B.W. Drinkwater, J. Sound Vibrat. 329, 4279 (2010).

[28] J.M. Nichols, C.C. Olson, J. Sound Vibrat. 329, 1165 (2010).

[29] H. Gökdag, O. Kopmaz, J. Sound Vibrat. 324, 1158 (2009).

[30] U. Jung, B.-H. Koh, J. Sound Vibrat. 321, 590 (2009).

[31] K.V. Nguyen, H.T. Tran, J. Sound Vibrat. 329, 4455 (2010).

[32] W. Staszewski, C. Boller, G. Tomlinson, Health Monitoring of Aerospace Structures, Wiley, Chichester 2004.

[33] G. Baker, J. Gollub, Chaotic Dynamics: An Introduction, Cambridge University Press, Cambridge 1990.

[34] W. Batko, L. Majkut, Arch. Metal. Mater. 52, 389 (2007).
[35] W. Batko, L. Majkut, Arch. Metal. Mater. 55, 757 (2010).

[36] L. Majkut, Biuletyn Wojskowej Akademii Technicznej (Military University of Technology) 59, 181 (2010) (in Polish).

[37] I. Trendafilova, E. Manoach, Mech. Syst. Sign. Proc. 22, 1092 (2008).

[38] H. Abarbanel, Analysis of Observed Chaotic Data, Springer, New York 1996

[39] H. Schuster, Deterministic Chaos: An Introduction, Wiley, Weinheim 1995.

[40] J. Nichols, M. Seaver, S. Trickey, J. Sound Vibrat. 297, 1 (2006).

[41] D. Boomehead, P. King, Physica D 20, 217 (1986).

[42] J.B. Elsner, A.A. Tsonis, Singular Spectrum Analysis: A New Tool in Time Series Analysis, Springer, New York 1996.

[43] N. Pakard, J. Crutchfield, J. Farmer, R. Shaw, Phys. Rev. Lett. 45, 712 (1980).

[44] L. Majkut, J. Theor. Appl. Mech. (Warsaw) 47, 193 (2009).

[45] A. Darpe, K. Gupta, A. Chawla, J. Sound Vibrat. 269, 33 (2004).

[46] H. Nahvi, M. Jabbari, Int. J. Mech. Sci. 47, 1477 (2005).

[47] L. Majkut, Vibroacoustic diagnostics of structural components, Publishing House of Institute for Sustainable Technologies, Radom 2010 (in Polish). 